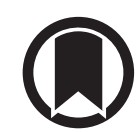

CrossMark

\section{Aspergillus-induced pneumonia in adult without obvious immunodeficiency: test the burst!}

\author{
To the Editor:
}

Chronic granulomatous disease (CGD) is a rare primary immunodeficiency caused by a germline defect in one of the genes (CYBB, NCF1, CYBA, NCF2) encoding the NADPH oxidase 2 (NOX2) components responsible for the phagocyte oxidative burst and the production of reactive oxygen species (ROS). An early diagnosis and a prompt treatment are crucial for improving outcomes in affected patients. CGD is usually diagnosed in early childhood, and more than $95 \%$ of cases are diagnosed by the age of 5 years $[1$, 2]. However, CGD can be diagnosed late in adulthood [1-3]. Aside from residual NOX2 activity, factors underlying late diagnosis of CGD remain poorly investigated. Here, we report a case of Aspergillus-induced pneumonia rapidly extending to fatal acute respiratory distress syndrome (ARDS) revealing a late diagnosis of CGD, which was associated with a residual mitochondria-driven neutrophil extracellular trap release (NETosis) counterbalancing the lack of functional NOX2. Written informed consent was obtained from the deceased patient's next-of-kin for publication of this case report and accompanying images.

A previously healthy 24-year-old Tibetan native man presented at the emergency room (day 1) with fever, cough and shortness of breath worsening over 5 days. No treatment or evidence for an immunocompromised state was reported in the past medical history. The initial chest radiograph revealed bilateral infiltrates. After 4 days of outpatient treatment with macrolides, the respiratory failure worsened leading to urgent endotracheal intubation and mechanical ventilation support at day 5. The chest radiograph demonstrated extensive bilateral alveolar infiltrates. A diagnosis of severe ARDS was made [4]. Despite the negativity of all bacteriological samples and given the high suspicion of pneumonia, broad-spectrum antibiotic therapy was started at day 5. Extensive diagnostic workup on bronchoalveolar lavage (BAL) and blood samples (standard microbiology and virology, universal PCR, immunostaining and immunofluorescence, and screening for autoimmune diseases) remained negative, except for Aspergillus fumigatus with positive PCR in BAL. Intravenous voriconazole was started at day 10. In addition to lung failure, the patient developed septic shock and acute renal failure at day 13. Because of refractory hypoxaemia a veno-venous extracorporeal membrane oxygenator (ECMO) was placed at day 13 and the patient was therefore transferred to our ECMO referral centre. Chest computed tomography imaging revealed bilateral alveolar-interstitial infiltrates centred around the bronchial tree (figure 1a), compatible with a bronchogenic dissemination of an infectious pathogen. These ground-glass patterns also coexisted with multiple excavated pulmonary nodules in the anterior parts of the chest. Culture of BAL fluid samples were positive for Aspergillus fumigatus. As we did not expect Aspergillus spp.-induced ARDS in an immunocompetent patient, we performed a lung biopsy at day 18. Histological findings showed mycelial filaments with neutrophils accumulating in disseminated granuloma.

Due to a suspected neutrophil defect, an extended ex vivo functional analysis was performed enabling the diagnosis of CGD [5]. While neutrophil chemotaxis and phagocytosis were normal (data not shown), the nitro-blue tetrazolium reduction test showed a dramatically impaired ROS production (figure $1 \mathrm{~b}$ ), which was confirmed by luminol-amplified chemiluminescence and cytochrome $\mathrm{c}$ reduction assays (figure 1c and d). The five components of NOX2 were detectable (figure 1e), suggesting the presence of a mutation allowing protein expression, but strongly impairing enzymatic activity. This is in line with some residual NOX2 activity (data not shown). Subsequently, DNA sequence analysis by fluorescent sequencing revealed a hemizygous missense mutation in exon 10 of the $C Y B B$ gene encoding gp91phox subunit (NCBI

@ERSpublications

Unusual pathogen-driven sepsis should prompt to screen for chronic granulomatous disease, even in adult patients http://ow.ly/2FSi30iXUsa

Cite this article as: Flament $\mathrm{H}$, Granger V, Vezinet $\mathrm{C}$, et al. Aspergillus-induced pneumonia in adult without obvious immunodeficiency: test the burst!. Eur Respir J 2018; 51: 1702711 [https://doi.org/ 10.1183/13993003.02711-2017]. 


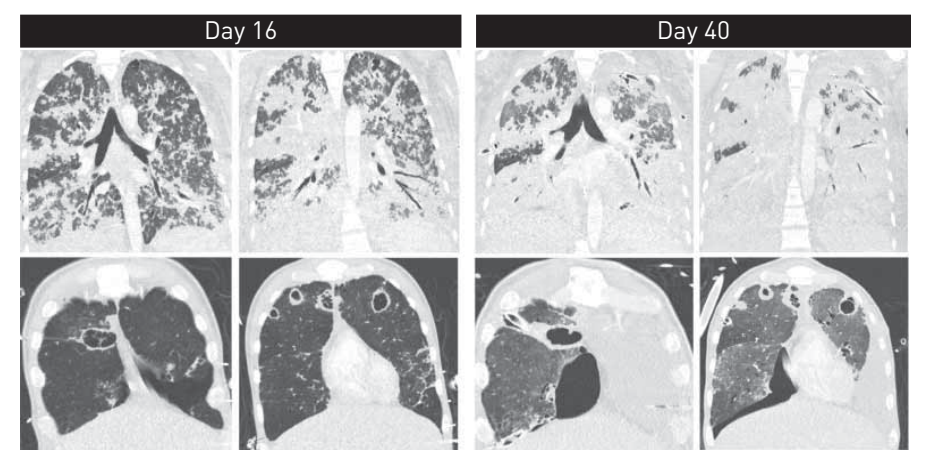

e)

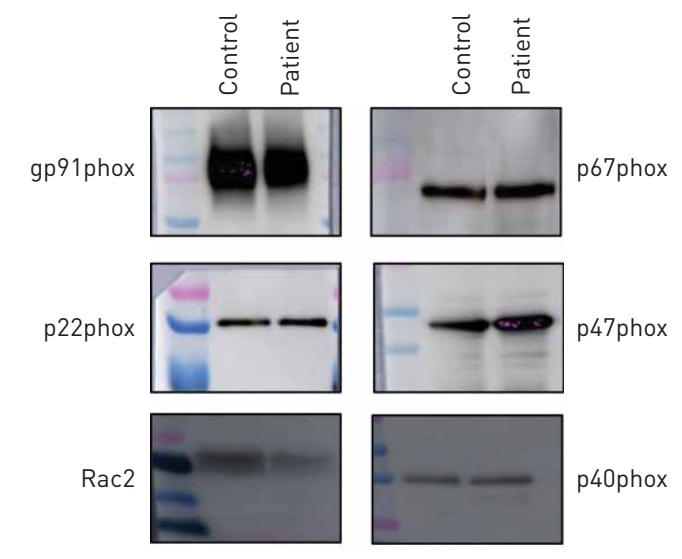

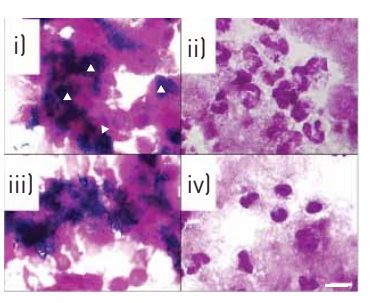
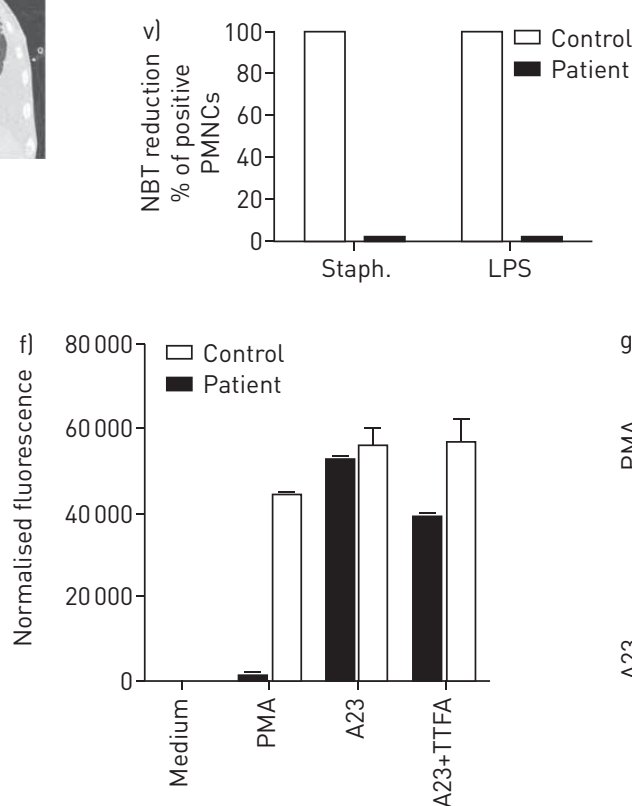
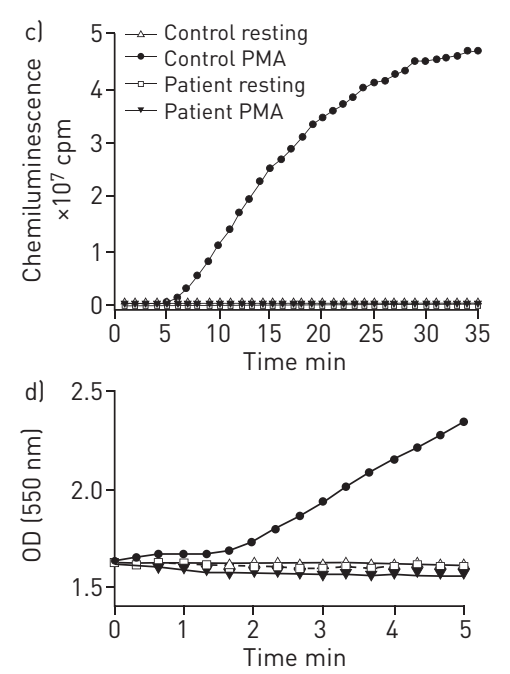

g)

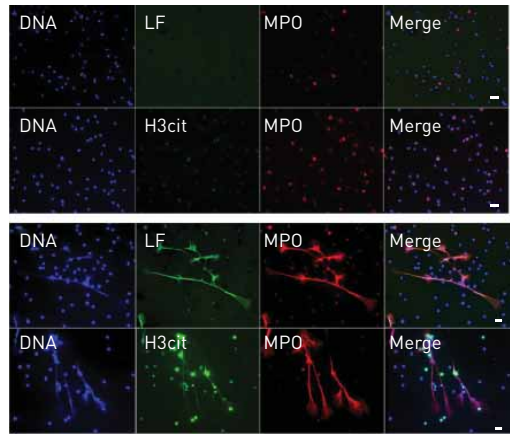

FIGURE 1 Elements of the chronic granulomatous disease (CGD) diagnosis. a) Computed tomography imaging at day 16 displaying bilateral extensive alveolar-interstitial infiltrates centred around the bronchial tree. These ground-glass patterns also coexisted with multiple excavated pulmonary nodules in the anterior parts of the chest. Overall bilateral lesions eventually worsened, shown in the computed tomography imaging at day 40. b) Comparison of nitro-blue tetrazolium (NBT) reduction in neutrophils isolated from a healthy donor and the CGD patient. Whole blood was incubated for $20 \mathrm{~min}$ at $37^{\circ} \mathrm{C}$ in the presence of NBT with either serum-opsonised coagulase-negative Staphylococci (Staph.) (i) control and ii) CGD patient) or lipopolysaccharide (LPS) (iii) control and iv) CGD patient). Following formol fixation and red blood cell lysis, leukocytes were spun onto a microscope slide using a cytocentrifuge and stained with fuchsin. v) Quantification of NBT reduction was performed by measuring the percentage of neutrophils with intracellular NBT formazan deposits (white arrow heads in i). Scale with fuchsin. v) Quantification of NBT reduction was performed by measuring the percentage of neutrophils with intracellular NBT formazan deposits (white arrow heads in i). Scale
bar $=20 \mu \mathrm{m}$. c) Comparison of reactive oxygen species (ROS) production by neutrophils isolated from a healthy donor and the CGD patient. Neutrophils were purified using Dextran/Ficoll technique. Neutrophils $\left(0.5 \times 10^{5}\right.$ cells per $\left.0.5 \mathrm{~mL}\right)$ were incubated in Hank's buffer for 10 min in the presence of luminol $(10 \mu \mathrm{M})$ at $37^{\circ} \mathrm{C}$. ROS production was measured after stimulation with phorbol myristate acetate (PMA) (100 $\left.\mathrm{ng} \cdot \mathrm{mL}^{-1}\right)$ using a chemiluminometer. d) Comparison of superoxide anion production by neutrophils isolated from a healthy donor and the CGD patient. Neutrophils $\left(\times 10^{6}\right.$ cells per $\left.\mathrm{mL}\right)$ were preincubated for $10 \mathrm{~min}$ at $37^{\circ} \mathrm{C}$ with cytochrome $\mathrm{C}\left(1 \mathrm{mg} \cdot \mathrm{mL}^{-1}\right)$ in HBSS and stimulated with PMA $\left(100 \mathrm{ng} \cdot \mathrm{mL}^{-1}\right)$. Reduction of cytochrome $\mathrm{C}$ was determined by measuring optical density $10 \mathrm{D}$ ) t $550 \mathrm{~nm}$ in an UVIKON 860 spectrophotometer. Immunoblot analysis of NADPH oxidase 2 subunits in the CGD patient's neutrophils. 2phox, Rac2, p67phox, p47phox and anti-p40phox antibodies. F Extracellular DNA release from highty purified patient and control neutrophils (Miltenyi neutrophil isolation kit) as measured by Sytox Green fluorescence. Cells were stimulated with $25 \mathrm{nM} \mathrm{PMA,} 5 \mu$ M A23187 (A23) with or without thenoyltrifluoroacetone (TTFA) (10 $\mu$ M). Results were expressed as fluorescence arbitrary units normalised according to baseline and medium values using the following formula: (lstimulus fluorescence endpoint - stimulus fluorescence baseline) - (medium fluorescence endpoint - medium fluorescence baseline)). Data are shown as mean \pm SEM for each condition (run in triplicate). g) Immunostaining of neutrophil extracellular traps (NETs). Blood neutrophils isolated from the CGD patient were seeded on polylysine-coated glass coverslips, stimulated by PMA or A23187 (A23) and stained with anti-LF (green), anti-MPO (red) or anti-H3cit (green) antibodies. Co-staining of DNA used DAPI (blue) (magnification $\times 600$ ). Scale bar=20 $\mu$ M. LF: lactoferrin; MPO: myeloperoxidase; H3cit: citrullinated histone 3; PMNC: polymorphonuclear cell. 
reference sequence: NM_000397.3, c.1178C>T, p.Thr393Ile) (data not shown). This C-to-T nucleotide transition, which causes the change of threonine for isoleucine at position 393, has not been previously described in CGD and is not reported in exome sequencing data from more than 120000 individuals (gnomAD; http://gnomad.broadinstitute.org). This p.Thr393Ile substitution was predicted to have deleterious effects, since Thr393 is highly conserved in the NOX family enzymes. Indeed, Thr393 is located close to the NADPH binding site on the C-terminal part of the enzyme and this mutation might therefore impair NADPH binding and/or electron transfer to FAD.

Among the mechanisms highly involved in anti-fungal response, NETosis, i.e. the release of neutrophil extracellular traps (NETs) (extracellular DNA fibres decorated with intracellular antimicrobial components), has been highlighted [6]. NETs can ensnare and kill large pathogens, such as fungi, that are too large to be engulfed [7]. NETosis was assessed by quantifying extracellular DNA release as previously described [8]. While the calcium ionophore A23187, a NOX2-independent stimulus of NETosis, triggered the release of NETs from patient neutrophils ex vivo, phorbol myristate acetate, a NOX2-dependent stimulus of NETosis did not have any effect (figure 1f). Immunofluorescence confirmed that myeloperoxidase, lactoferrin and citrullinated histone 3 were only observed on DNA filaments in response to A23187 (figure 1g). Thenoyltrifluoroacetone, an inhibitor of the mitochondrial respiratory chain, decreased A23187-derived NETs suggesting a role for mitochondria in NOX2-independent NETosis (figure 1f). We thus confirm that the absence of functional NOX2 may force neutrophils to use mitochondria as an alternative ROS source, promoting mitochondria-driven NETosis, as already suggested [9]. Despite the eradication of Aspergillus from BAL fluid, the patient died at day 49 from a multiple organ failure with refractory ARDS.

CGD is usually diagnosed in early childhood. The mean age of diagnosis for the X-linked recessive form is 3 years of age, while the autosomal recessive forms are diagnosed at 7.8 years of age [1-3]. Few CGD cases have been diagnosed in adulthood beyond the fourth decade [1-3]. The vast majority of these late diagnosed cases were explained by some residual cytochrome b and ROS production. To our knowledge, there are no delayed cases of CGD linked to a residual neutrophil NETosis in the literature to date. The originality of our CGD case report, therefore, resides in the fact that the delayed manifestation of severe sepsis might be explained, at least in part, by the existence of residual mitochondria-driven NETosis due to mitochondrial-derived ROS. This form of NETosis, independent of NOX2 and not preponderant in the cell, could partly counterbalance the lack of functional NOX2 and participate in pathogen killing. However, despite the existence of this particular form of NETosis, the patient could not control his aspergillosis. GAZENDAM et al. [10] already suggested that CGD neutrophils could form NETs in response to Aspergillus hyphae, but could not kill them. Moreover, NETs inhibition effects on Aspergillus hyphae activity has been shown to be impaired in a CGD patient and restored after gene therapy [11]. In addition to being ineffective to clear infection, NETs might have worsened organ failure and contributed to the poor patient outcome. Indeed, their capacity to damage tissues is particularly well documented in sepsis [12], acute lung injury [13] and an experimental model of influenza-induced ARDS [14].

Like other fungi or parasites, such as Pneumocystis jirovecii, Aspergillus was identified as a pathogen causing ARDS in immunosuppressed patients [15-17]. Every time the diagnostic workup identifies such pathogens as the sole cause of ARDS, clinicians should screen for either a primary or secondary acquired immunocompromised state, i.e. isolated or combined humoral and cellular immunity, complement proteins, or phagocytic cell disorders including CGD. Moreover, we suggest that adults presenting with a life-threatening sepsis related to unusual pathogens, in the absence of obvious secondary immunodeficiency (e.g. diabetes mellitus or corticosteroid use) should also be screened for underlying immunodeficiency including CGD. Early diagnosis of CGD remains crucial, since benefits can result from haematopoietic stem cell transplantation, infection prophylaxis and genetic counseling for the relatives.

Héloïse Flament ${ }^{1,2,10}$, Vanessa Granger ${ }^{3,4,10}$, Corinne Vezinet ${ }^{5}$, Viviana Marzaioli ${ }^{2}$, Caroline Kannengiesser $\odot^{6}$, Luc de Chaisemartin ${ }^{3,4}$, Margarita Hurtado-Nedelec ${ }^{1,2}$, Elena Litvinova ${ }^{1}$, Jonathan Messika ${ }^{7,8,9}$, Nicolas Adam ${ }^{5}$, Marie-Anne Gougerot-Pocidalo ${ }^{1,2}$, Pham My-Chan Dang ${ }^{4}$, Renato Monteiro ${ }^{1,2}$, Jamel el Benna ${ }^{2}$, Olivier Langeron ${ }^{5}$, Sylvie Chollet-Martin ${ }^{3,4}$ and Antoine Monsel $\odot^{5}$

${ }^{1}$ Assistance Publique des Hôpitaux de Paris, Laboratoire d'immunologie " Dysfonctionnements Immunitaires », Hôpital Bichat, Paris, France. ${ }^{2}$ INSERM-U1149, CNRS-ERL8252, Centre de Recherche sur l'Inflammation, Université Paris Diderot, Sorbonne Paris Cité, Laboratoire d'Excellence Inflamex, DHU FIRE, Faculté de Médecine, Site Xavier Bichat, Paris, France. ${ }^{3}$ Inflammation Chimiokines et Immunopathologie, INSERM, Fac. de pharmacie, Univ. Paris-Sud, Université Paris-Saclay, Châtenay-Malabry, France. ${ }^{4}$ Assistance Publique des Hôpitaux de Paris, Laboratoire d'immunologie "Autoimmunité et Hypersensibilités», Hôpital Bichat, Paris, France. ${ }^{5}$ Réanimation Chirurgicale Polyvalente, Département d'Anesthésie-Réanimation, Hôpital Pitié-Salpêtrière, Assistance Publique des Hôpitaux de Paris, Médecine Sorbonne Université, Paris, France. ${ }^{6}$ Assistance Publique des Hôpitaux de Paris, laboratoire de génétique, Hôpital Bichat, Paris, France. ${ }^{7}$ Assistance Publique des Hôpitaux de Paris, Hôpital Louis Mourier, Service de Réanimation Médico-Chirurgicale, Colombes, France. ${ }^{8}$ INSERM, IAME, U1137, Paris, France. ${ }^{9}$ Université Paris Diderot, Sorbonne Paris Cité, IAME, UMRS 1137, Paris, France. ${ }^{10}$ Both authors contributed equally. 
Correspondence: Antoine Monsel, Anesthesia and Critical Care Dept, CHU Pitié-Salpêtrière, 47-83, Boulevard de l'Hôpital, 75013 Paris, France. E-mail: antoine.monsel@aphp.fr

Received: Dec 282017 | Accepted after revision: Feb 192018

Acknowledgments: We thank Benoît Noël (Unité mixte de Recherche 996-Inflammation, Chemokines and Immunopathology, Institut National de la Santé et de la Recherche Médicale, Université Paris Sud, Université ParisSaclay, Châtenay-Malabry, France) and Claire Oudin (AP-HP, Centre Hospitalier Universitaire Xavier Bichat, Service d'Immunologie et d'Hématologie et Service de Biochimie Hormonale et Génétique, CIB Phenogen, Paris F-75018, France) (molecular exploration of $C Y B B$ ) for excellent technical help.

Conflict of interest: None declared.

\section{References}

1 Dunogue B, Pilmis B, Mahlaoui N, et al. Chronic granulomatous disease in patients reaching adulthood: a nationwide study in France. Clin Infect Dis 2017; 64: 767-775.

2 Salvator $\mathrm{H}$, Mahlaoui N, Catherinot $\mathrm{E}$, et al. Pulmonary manifestations in adult patients with chronic granulomatous disease. Eur Respir J 2015; 45: 1613-1623.

3 Colin de Verdiere S, Noel E, Lozano C, et al. Respiratory complications lead to the diagnosis of chronic granulomatous disease in two adult patients. J Clin Immunol 2017; 37: 113-116.

4 Force ADT, Ranieri VM, Rubenfeld GD, et al. Acute respiratory distress syndrome: the Berlin definition. JAMA 2012; 307: 2526-2533.

$5 \quad$ Roos D. Chronic granulomatous disease. Br Med Bull 2016; 118: 50-63.

6 Sorensen OE, Borregaard N. Neutrophil extracellular traps - the dark side of neutrophils. J Clin Invest 2016; 126: $1612-1620$.

7 Branzk N, Lubojemska A, Hardison SE, et al. Neutrophils sense microbe size and selectively release neutrophil extracellular traps in response to large pathogens. Nat Immunol 2014; 15: 1017-1025.

8 Granger V, Faille D, Marani V, et al. Human blood monocytes are able to form extracellular traps. J Leukoc Biol 2017; 102: 775-781.

9 Lood C, Blanco LP, Purmalek MM, et al. Neutrophil extracellular traps enriched in oxidized mitochondrial DNA are interferogenic and contribute to lupus-like disease. Nat Med 2016; 22: 146-153.

10 Gazendam RP, van Hamme JL, Tool AT, et al. Human neutrophils use different mechanisms to kill Aspergillus fumigatus conidia and hyphae: evidence from phagocyte defects. J Immunol 2016; 196: 1272-1283.

11 Bianchi M, Niemiec MJ, Siler U, et al. Restoration of anti-Aspergillus defense by neutrophil extracellular traps in human chronic granulomatous disease after gene therapy is calprotectin-dependent. J Allergy Clin Immunol 2011; 127: $1243-1252$.

12 Czaikoski PG, Mota JM, Nascimento DC, et al. Neutrophil extracellular traps induce organ damage during experimental and clinical sepsis. PLoS One 2016; 11: e0148142.

13 Liu S, Su X, Pan P, et al. Neutrophil extracellular traps are indirectly triggered by lipopolysaccharide and contribute to acute lung injury. Sci Rep 2016; 6: 37252.

14 Narasaraju T, Yang E, Samy RP, et al. Excessive neutrophils and neutrophil extracellular traps contribute to acute lung injury of influenza pneumonitis. Am J Pathol 2011; 179: 199-210.

15 Jindal AK, Rawat A, Suri D, et al. Severe Aspergillus pneumonia and pulmonary artery hypertension in a child with autosomal recessive chronic granulomatous disease and selective IgA deficiency. J Clin Immunol 2017; 37: 333-335.

16 Papazian L, Calfee CS, Chiumello D, et al. Diagnostic workup for ARDS patients. Intensive Care Med 2016; 42: 674-685.

17 Segal BH, Romani LR. Invasive aspergillosis in chronic granulomatous disease. Med Mycol 2009; 47: Suppl. 1, S282-S290. 